\title{
Measurement of the Exposed Eyeball Area and Vertical Dimension of Palpebral Fissure after Double Eyelidplasty
}

\author{
Seung Hun Yeo, Dong Gil Han \\ Department of Plastic and Reconstructive \\ Surgery, Catholic University of Daegu, \\ School of Medicine, Daegu, Korea
}

No potential conflict of interest relevant to this article was reported.
Background Although the technique of double eyelidplasty is well recognized, there have been a few reports on the measurement of the eyelid after the operation. Herein, we measured the changes of the eyelids photogrammatically and compared them with others.

Methods Database of patients who had undergone double eyelidplasty using a threepartial-incision technique, along with a follow-up of more than 6 months, were identified. The changes of eyelid were measured by exposed eyeball area (EEA) and vertical dimension of palpebral fissure (VDPF) using preoperative and postoperative photographs.

Results Between January 2012 and December 2013, there were 28 patients who were followed-up for more than 6 months. All patients were female between the age of 16 and 37 years; the average follow-up period was 11.4 months. The mean preoperative value of EEA was $74.6 \pm 9.1 \%$ and that of VDPF was $7.4 \pm 1.0 \mathrm{~mm}$. Conversely, the mean postoperative value of EEA was $83.9 \pm 6.3 \%$ and that of VDPF was $8.5 \pm 1.0 \mathrm{~mm}$. Conclusions The mean values of EEA and VDPF after double eyelidplasty had changed with statistical significance.

Keywords Blepharoplasty, Eyelids, Outcomes

\section{INTRODUCTION}

It has been reported that as many as $50 \%$ of normal young Korean females have only a single eyelid or an unrecognizable supratarsal fold $[1,2]$. The ultimate purpose of a double-eyelid operation is to construct a durable, natural-looking supratarsal fold with predictable, long-lasting results [3,4]. Recently, the partial- or limited-incision method has become more and more popular with advancements in surgical techniques [4-7].

Received: Nov 21, 2016 Revised: Jan 10, 2017 Accepted: Jan 11, 2017 Correspondence: Dong Gil Han Department of Plastic and Reconstructive Surgery, Daegu Catholic University Medical Center, 33 Duryugongwon-ro 17-gil, Nam-gu, Daegu 42472, Korea. E-mail: dghan1001@cu.ac.kr

Copyright @ 2017 The Korean Society for Aesthetic Plastic Surgery.

This is an Open Access article distributed under the terms of the Creative Commons Attribution Non-Commercial License (http://creativecommons.org/licenses/by-nc/4.0/) which permits unrestricted non-commercial use, distribution, and reproduction in any medium, provided the original work is properly cited. www.e-aaps.org
However, even though various double-eyelid techniques are well recognized, there remains a lack of reviews or reports on the qualitative outcomes of double eyelidplasty. In addition, previous reports of eyelid dimensions based on anthropometric or photogrammetric studies of Koreans have not addressed whether the subjects had a double eyelid. Therefore, the values have varying ranges according to the number of subjects with double eyelids. In view of this, we compared the preoperative and postoperative values of the eyelids following double-eyelid operations. To control for the outcomes, a comparison was made between these values and the previously reported anthropometric and photogrammetric values of normal young Korean females.

\section{METHODS}

\section{Patient evaluation}

The database of patients who had undergone double eyelidplasty with a 3-part incision between January 2012 and December 2013 
was reviewed; we included 56 eyelids from 28 patients who were without any disease or any history of eyelid surgery and who were followed up for more than 6 months. The clinical evaluation included patient age, sex, follow-up period, complications, and objective changes to the eyelid, both preoperatively and postoperatively, measured by exposed eyeball area (EEA) and the vertical dimension of the palpebral fissure (VDPF).

The EEA was examined using a Java-based image-processing program (Image J 1.40; National Institutes of Health, Bethesda, MD, USA). EEA is defined as the percentage of the exposed area of the cornea and sclera taken up by the theoretically maximal exposed area of the cornea and sclera between the lower lid margin at the lowest margin of the limbus and the upper lid margin at the highest margin of the limbus [8] (exposed area/theoretically maximal exposed area $\times 100$ ) (Fig. 1). The VDPF, based on corneal diameter, was determined using photographs of each patient taken before and after surgery and measuring with the ruler tool in Adobe Pho- toshop CS3 (Adobe Systems, San Jose, CA, USA). To minimize any error caused by size differences of the corneal diameter, we performed measurements and corrected our findings to match the average corneal diameter $(11.3 \mathrm{~mm})$ of Korean females [9]. In addition, we attempted to photograph subjects when their forehead muscles were at rest, in order to prevent size differences in the eyelids (Fig. 2).

Statistical analysis was performed using SPSS Statistics 19.0 (IBM Corp., Armonk, NY, USA). A comparison between the preoperative and postoperative values was carried out using a paired t-test. All P-values of less than 0.05 were considered to indicate statistical significance. In addition, the changes to the EEA of the pseudoptosis group were compared against those of the normal-eyelid group.

\section{Surgical techniques}

The desired fold line for the double eyelid was noted with the patient in sitting position, and the location of the 3-part incision was

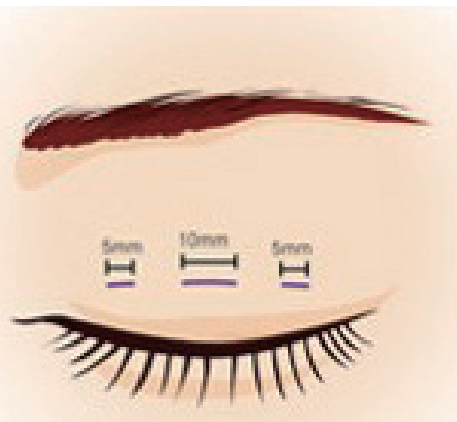

(A)

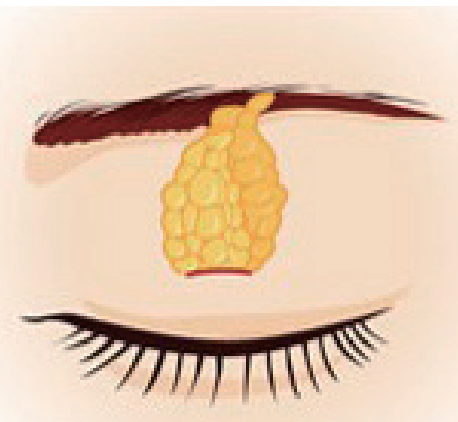

(B)

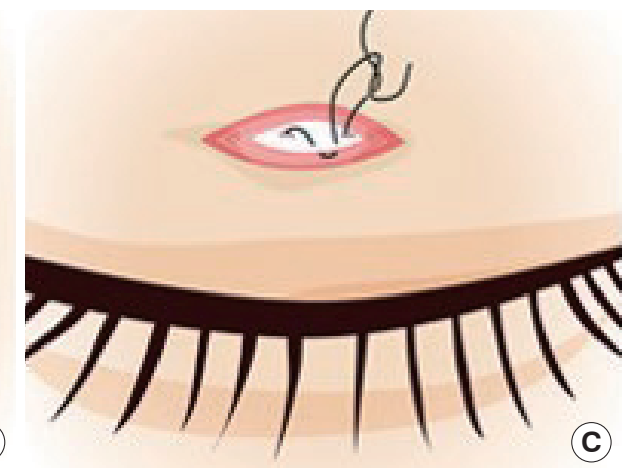

Fig. 1. Operative procedures of three-partial-incision double eyelidplasty. (A) Preoperative design. The designed incision line, approximately 10 $\mathrm{mm}$ in length, is marked on the central one-third of the eyelid, and marked with a length of $5 \mathrm{~mm}$ to the medial and lateral one-third. (B) Through the central incision, the anterior septum is opened and the orbital fat is resected. (C) Tarso-dermomuscular horizontal mattress suture is placed using 7-0 white nylon.
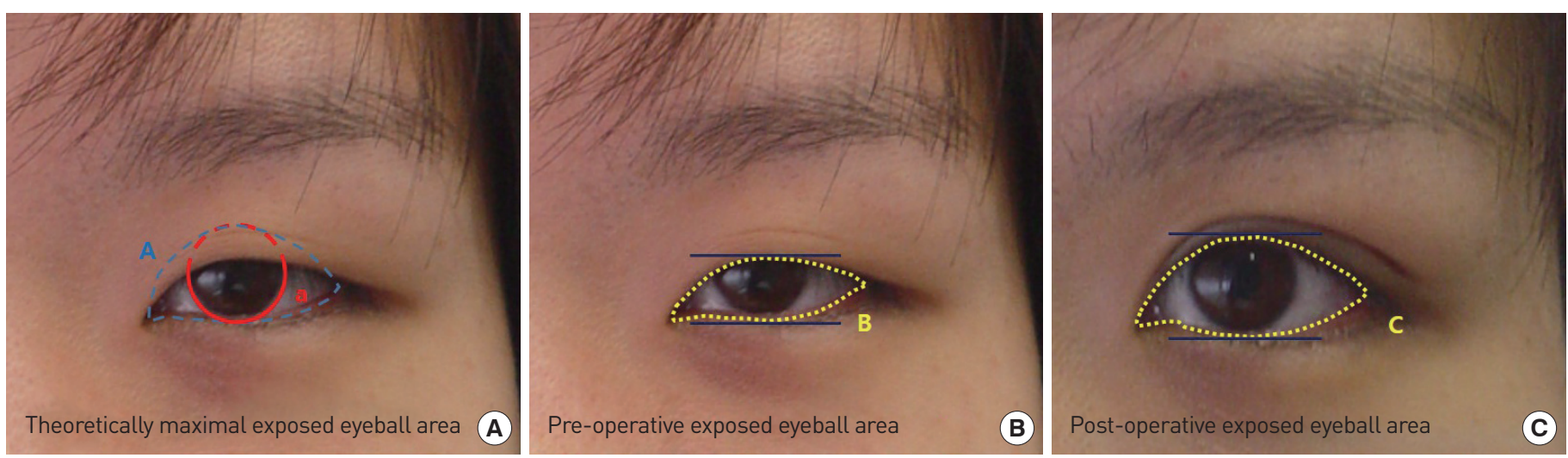

Fig. 2. Measurement of exposed eyeball area. (A) Totally theoretically exposed cornea \& sclera area between lower lid margin at lowest margin of limbus and upper lid margin at highest margin of limbus. (B) Preoperative grossly visible cornea \& sclera area. (C) Postoperative grossly visible cornea \& sclera area. A) Theoretically maximal exposed eyeball area. B) Pre-operative exposed eyeball area. C) Post-operative exposed eyeball area. ${ }^{\text {al }}$ Margin of limbus. 

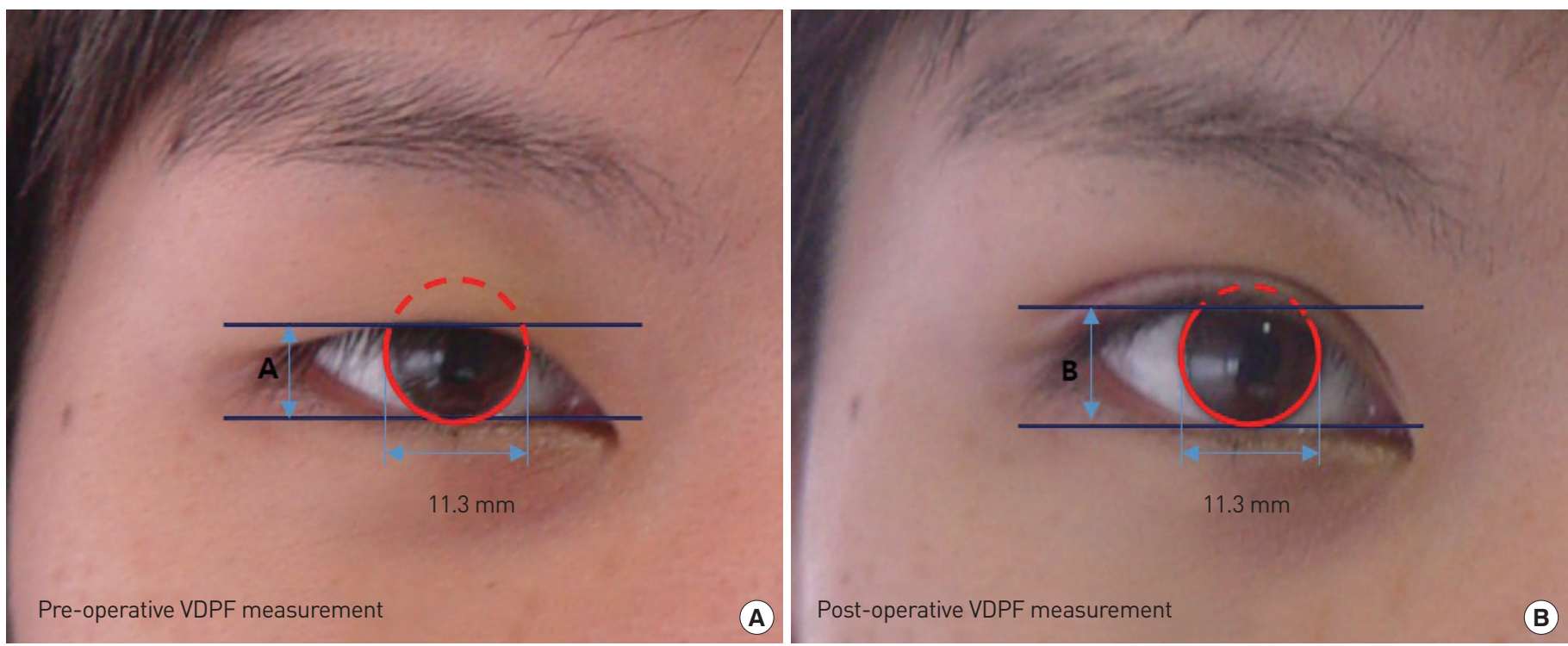

Fig. 3. Measurement of pre- and postoperative vertical dimension of palpebral fissure. (A) The distance between the upper and lower eyelid in vertical alignment with the center of the pupil in primary gaze at preoperative photo $(7.9 \mathrm{~mm})$. (B) The distance between the upper and lower eyelid in vertical alignment with the center of the pupil in primary gaze, a postoperative photo (9.8 $\mathrm{mm}$ ).

decided. According to the design, an approximately 10 -mm incision line was marked on the central one-third of the eyelid, and a small 5-mm incision line was marked on either side of the central incision on both the medial and lateral one-third of the eyelid.

All patients underwent surgery under local anesthesia using 1\% lidocaine hydrochloride ( $\mathrm{HCl}$ ) combined with 1:100,000 epinephrine. Subsequently, through a central incision, the orbicularis oculi muscle was dissected in a perpendicular direction. The pretarsal fat layer was then spread with scissors to expose the tarsal plate's upper border. Then, the anterior orbital septum was pinched out with fine Adson forceps and opened, and the herniated orbital fat was delivered and resected. The remnant stalks of orbital fat were tied with 6-0 polydioxanone (PDS) to prevent hematoma. The skinto-tarsal fixation suture was passed horizontally on the levator aponeurosis or tarsus with 7-0 white nylon and plicated to the dermomuscular margin and orbicularis oculi muscle of the inferior skin incision. The patient was then asked to open her eyes, and the fold height and shape were checked, and then the fixation suture was tied permanently. The same procedure was also performed on the opposite eyelid. The patient was then asked to open her eyes repeatedly to observe and to adjust the supratarsal crease to achieve bilateral symmetry. The same skin-to-tarsal fixation with 7-0 nylon was performed through small medial and lateral incisions on both upper eyelids. After achieving a bilateral symmetry of the created crease, a skin suture was placed with a 7-0 black nylon (Fig. 3).

\section{RESULTS}

All 28 patients were young females between the ages of 16 and 37
Table 1. Patient demographics

\begin{tabular}{lc}
\hline Characteristics & No. of patients $(\mathbf{n}=\mathbf{2 8})$ \\
\hline No. of eyelids & 56 \\
Age & $22 \pm 6.1$ \\
Sex & \\
Female & $28(100.0)$ \\
Male & $0(0.0)$ \\
Follow-up & $11.3 \pm 12.0$ \\
\hline
\end{tabular}

The data is presented as mean \pm standard deviation or number (\%).

Table 2. Postoperative complications

\begin{tabular}{lc}
\hline Complications & No. of patients $(\mathbf{n}=28)$ \\
\hline Supratarsal loosening & $2(7.1 \%)$ \\
Conjunctivitis & $1(3.6 \%)$ \\
Fold asymmetry & $0(0.0 \%)$ \\
Abnormal eyelid contour & $0(0.0 \%)$ \\
Hematoma & $0(0.0 \%)$ \\
Total & $3(10.7 \%)$ \\
\hline
\end{tabular}

The data is presented as number (\%).

years, with a mean age of $22 \pm 6.1$ years. The average follow-up period was 11.4 months, and the longest follow-up lasted for 48 months. Within 1 to 2 weeks, the postoperative edema had mostly subsided, and a more natural-looking double eyelid was evident within 2 weeks to 1 month postoperatively (Table 1). All patients were satisfied with the surgical outcome. However, one complication of conjunctivitis 
Table 3. Objective outcome of total group comparison of the pre- and postoperative values

\begin{tabular}{lrcc}
\hline $\begin{array}{l}\text { Objective outcomes } \\
(\%, \mathrm{~mm})\end{array}$ & Preoperation & Postoperation & P-value $^{\text {a) }}$ \\
\hline EEA & $74.6 \pm 9.1$ & $83.9 \pm 6.3$ & $<0.001$ \\
VDPF & $7.4 \pm 1.0$ & $8.5 \pm 1.0$ & $<0.001$
\end{tabular}

The data is presented as mean \pm standard deviation.

EEA, exposed eyeball area; VDPF, vertical dimension of palpebral fissure.

alPaired t-test.

Table 4. The changes of exposed eyeball area of pseudoptosis group (n = 17)

\begin{tabular}{lcc}
\hline No. & Preoperation, EEA $(\%)$ & Postoperation, EEA $(\%)$ \\
\hline 1 & 68.0 & 76.0 \\
2 & 52.0 & 84.0 \\
3 & 79.0 & 91.0 \\
4 & 71.0 & 83.5 \\
5 & 61.0 & 67.0 \\
6 & 67.0 & 81.0 \\
7 & 79.0 & 83.0 \\
8 & 83.0 & 88.0 \\
9 & 73.0 & 80.0 \\
10 & 81.0 & 85.0 \\
11 & 63.0 & 76.0 \\
12 & 70.0 & 87.0 \\
13 & 70.0 & 88.0 \\
14 & 54.0 & 78.0 \\
15 & 82.0 & 89.0 \\
16 & 71.0 & 74.5 \\
17 & 86.0 & 87.0 \\
Average (mean \pm SD & $71.2 \pm 9.8$ & $82.2 \pm 6.3$ \\
\hline
\end{tabular}

EEA, exposed eyeball area; SD, standard deviation.

occurred, and unilateral supratarsal crease loosening was observed in 2 patients (Table 2).

The mean preoperative EEA of all patients was $74.6 \pm 9.1 \%$, and the average preoperative VDPF was $7.4 \pm 1.0 \mathrm{~mm}$. The mean postoperative EEA of all patients was $83.9 \pm 6.3 \%$, and the average postoperative VDPF was $8.5 \pm 1.0 \mathrm{~mm}$. In this study, patients showed a mean increase in EEA of $9.3 \%$ and a mean increase in VDPF of $14.9 \%$. The mean postoperative EEA and VDPF of all patient values showed statistically significant changes compared with the preoperative EEA and VDPF values $(\mathrm{P}=0.000)$ (Table 3$)$. The mean preoperative EEA of the pseudoptosis group was $71.2 \pm 9.8 \%$, and the mean postoperative EEA of the pseudoptosis group was $82.2 \pm 6.3 \%$ (Table 4). The mean preoperative EEA of the normal-eyelid group was $80.0 \pm 4.1 \%$, and the mean postoperative EEA of the normaleyelid group was $86.5 \pm 5.4 \%$ (Table 5 ). The corresponding postop-
Table 5. The changes of exposed eyeball area of normal eyelid group ( $n=11)$

\begin{tabular}{lcc}
\hline No. & Preoperation, EEA (\%) & Postoperation, EEA (\%) \\
\hline 1 & 78.0 & 84.0 \\
2 & 79.0 & 82.5 \\
3 & 85.5 & 92.0 \\
4 & 87.0 & 90.5 \\
5 & 76.0 & 81.0 \\
6 & 81.0 & 82.0 \\
7 & 77.0 & 88.0 \\
8 & 75.0 & 77.5 \\
9 & 79.5 & 89.5 \\
10 & 78.5 & 89.5 \\
11 & 83.0 & 94.5 \\
Average (mean \pm SD) & $80.0 \pm 4.1$ & $86.5 \pm 5.4$ \\
\hline
\end{tabular}

EEA, exposed eyeball area; SD, standard deviation.

Table 6. Summary of studies of vertical dimension of palpebral fissure of Korean female youths

\begin{tabular}{lcc}
\hline Reference & VDPF & Double fold (\%) \\
\hline Lee et al., 1989 [15] & $7.88 \pm 0.8$ & - \\
Park et al., 2008 [12] & $8.2 \pm 1.1$ & 36 \\
Park et al., 1990 [13] & $8.04 \pm 1.55$ & 50 \\
Song et al., 2001 [2] & - & 36 \\
Moon et al. 2003 [17] & - & 48 \\
Song et al., 2002 [1] & $10.7 \pm 1.8$ & 100 \\
& $9.7 \pm 1.6$ & 0 \\
Bae et al., 2007 [9] & $8.2 \pm 1.6$ & 44.9 \\
Seo, 2009 [14] & $8.18 \pm 0.80$ & - \\
\hline
\end{tabular}

The data is presented as mean \pm standard deviation. VDPF, vertical dimension of palpebral fissure.

erative values of VDPF changed by a greater margin than the ranges for Korean female youths from previous reports, with about 50\% showing a double fold (Table 6).

\section{DISCUSSION}

East Asian eyelids (including those of Koreans) are characterized by pretarsal skin laxity, with fatty lids and the absence of a supratarsal fold produced by a lack of levator aponeurosis extension to the dermis of the pretarsal area $[9,10]$.

Various techniques to create a double eyelid in East Asians have been described, and they can be classified into 3 principal methods: non-incision (buried-suture), partial-incision, and full-incision techniques. Regardless of the chosen method, the surgical goal of double-eyelid operations is to achieve a durable, natural-looking 
fold while providing the shortest possible recovery time.

Currently, various partial- or limited-incision methods are in use worldwide. These limited- or partial-incision methods minimize tissue injury and postoperative edema, providing adequate adhesion for durable fold formation with a short recovery period. Previous reports have focused on surgical techniques and fold aspects, and there has been a general lack of reviews or reports on the objective qualitative outcomes of double eyelidplasty.

To evaluate the objective changes of the eyelids after a double eyelidplasty, EEA and VDPF were measured both preoperatively and postoperatively. In addition, EEA was measured according to the presence of pseudoptosis. In this study, when the marginal reflex distance-1 (MRD1) is $3 \mathrm{~mm}$ or less with normal levator function, it was defined as pseudoptosis [11]. Moreover, a comparison was made between these values and previously reported anthropometric and photogrammetric values in normal Korean youths.

In our hospital, a preliminary study of the values of EEA was performed on 90 subjects between the ages of 11 and 85 years, without any history of eyelid surgery or disease of the eyes, and the value was $74.0 \pm 8.1 \%$. This is similar to the preoperative EEA value in the current study $(74.6 \pm 9.1 \%)$.

For the anthropometric VDPF, Park et al. [12] reported a mean value of $8.2 \pm 1.1 \mathrm{~mm}$ in female subjects; among them, $36.1 \%$ of the individuals had double eyelids. In a different study, Park et al. [13] reported a mean value of $8.04 \pm 1.55 \mathrm{~mm}$ for VDPF in female subjects in their mid twenties; 50\% had double eyelids. Seo and Ahn [14] reported a mean value of $8.18 \pm 0.80 \mathrm{~mm}$ for VDPF in female subjects in their second decade; they did not provide the percentage of subjects who had double eyelids.

For photogrammetric VDPF, Song et al. [1] reported that the mean VDPF of subjects with double eyelids was $10.7 \pm 1.8 \mathrm{~mm}$ and those with single eyelids was $9.7 \pm 1.6 \mathrm{~mm}$; however, they did not know whether their subjects had undergone a double-eyelid operation. Lee et al. [15] reported a mean photogrammetric value of 7.88 $\pm 0.8 \mathrm{~mm}$ in young Korean females, but they did not mention the percentage of subjects with double eyelids. Bae et al. [9] reported a mean photogrammetric value of $8.2 \pm 1.6 \mathrm{~mm}$ in females in their $20 \mathrm{~s}$ and $8.2 \pm 1.7 \mathrm{~mm}$ in females in their 30s; among them, $44.9 \%$ of individuals had double eyelids.

In this study, the mean preoperative value of VDPF of all patients was $7.4 \pm 1.0 \mathrm{~mm}$, and the discrepancy with the other studies reported in the literature may have been due to the differences in the percentages of subjects who had double eyelids.

After the creation of a double eyelid, the postoperative values of EEA and VDPF for all patients $(83.9 \pm 6.3 \%$ and $8.5 \pm 1.0 \mathrm{~mm}$, respectively) showed statistically significant differences when compared with the preoperative values $(\mathrm{P}=0.000)$ (Table 3$)$. Meanwhile, some patients in the study had pseudoptosis due to upper-eyelid skin laxity. Therefore, we compared the values of the pseudoptosis group with those of the normal-eyelid group. The results showed that the changes in the EEA after double eyelidplasty in the pseudoptosis group (11\%) (Table 4) were greater than those in the normal-eyelid group (6.5\%) (Table 5), even though the absolute value of the normal-eyelid group was high postoperatively $(82.2 \%$ vs. $86.5 \%)$.

The mechanism of enlargement of the eyelid after double eyelidplasty is known to rely on the differences in thickness of tissue, the lower positioning of the orbit septum, and the prominent preaponeurotic fat resulting in a single full lid. Thus, after removal of the pretarsal fat and orbicularis muscle to form a dynamic connection or adhesion of the levator aponeurosis or tarsus and skin or dermis, pretarsal soft tissue is pulled up by adhesion with the tarsus upper margin and aponeurosis with a decrease of lid fullness. Additionally, when orbital fat is removed, the upper-eyelid burden decreases, which makes upper-eyelid excursion easier [16].

All patients were satisfied with the surgical outcomes, but there was 1 complication of conjunctivitis that required a removal of the buried suture. Moreover, 2 other patients experienced unilateral supratarsal crease loosening, which was corrected with an additional skin-to-tarsal fixation through the previous incision. During the early postoperative period, the eyes-closed view showed a slightly deep and intense double-eyelid line. As time passed, complaints of dry eye syndrome or changes involving tearing that could accompany an enlarged EEA did not occur. In addition, there were no occurrences of intense, unsatisfactory surgical scarring, and a naturallooking eyelid line was achieved.

The drawback of this study is that it included a small number of patients, which hinders the degree to which valid, generalizable conclusions can be drawn from our findings. Moreover, postoperative eyelid height can vary depending on whether the operative method is incisional or buried. Therefore, it is necessary to analyze and compare results according to the operative method.

\section{PATIENT CONSENT}

Patients provided written consent for the use of their images.

\section{REFERENCES}

1. Song WC, Park SH, Koh KS. Metric and non-metric characteristics of Korean eyes using standardized photographs. Korean J Phys Anthropol 2002;15:95-107.

2. Song WS, Kim YH, Lee SJ. Morphologic study of upper eyelid contour and functional evaluation of levator palpebrae superioris muscle in adult and young people. J Korean Ophthalmol Soc 2001;42:1523-9.

3. Hiraga Y. The double eyelid operation and augmentation rhinoplasty in the Oriental patient. Clin Plast Surg 1980;7:553-67.

4. Jinghe Z, Huifang X, Lihong W, et al. Three mini-incision double-eyelid blepharoplasty. Ann Plast Surg 2014;72:141-4.

5. Yang SY. Oriental double eyelid: a limited-incision technique. Ann Plast 
Surg 2001;46:364-8.

6. Lam SM, Kim YK. Partial-incision technique for creation of the double eyelid. Aesthet Surg J 2003;23:170-6.

7. Cho BC, Byun JS. New technique combined with suture and incision method for creating a more physiologically natural double-eyelid. Plast Reconstr Surg 2010;125:324-31.

8. Kim WJ, Park DH, Han DG. Ten years of results of modified frontalis muscle transfer for the correction of blepharoptosis. Arch Plast Surg 2016;43:172-80.

9. Bae TH, Kim JC, Kim WS, et al. A photogrammetic study of the eyes in Korean youths. J Korean Soc Plast Reconstr Surg 2007;34:37-43.

10. Bang YH. The double-eyelid operation without supratarsal fixation. Plast Reconstr Surg 1991;88:12-7; discussion 8-9.

11. Kim SK, Kim BG, Gum IS. Cosmetic \& reconstructive oculoplastic surgery. 3rd ed. Paju: Koonja; 2009.
12. Park DH, Choi WS, Yoon SH, et al. Anthropometry of Asian eyelids by age. Plast Reconstr Surg 2008;121:1405-13.

13. Park DM, Song JW, Han KW, et al. Anthropometry of Korean eyelids. J Korean Soc Plast Reconstr Surg 1990;17:822-41.

14. Seo HR, Ahn HB. Morphological changes of the eyelid according to age. J Korean Ophthalmol Soc 2009;50:1461-7.

15. Lee DJ, Kim WK, Chung CE. Photogrammetric study on the face of adult Korean female. J Korean Soc Plast Reconstr Surg 1989;16:42332.

16. Boo-Chai K. Plastic construction of the superior palpebral fold. Plast Reconstr Surg 1963;31:74-8.

17. Moon CS, Moon SH, Jang JW. Topographic anatomic difference of the eyelid according to age in Korean. J Korean Ophthalmol Soc 2003;44: 1865-71. 